\title{
Considerações estatísticas sobre os valores métricos dos segmentos corpóreos do sistema reprodutor dos escargots das espécies Achatina fulica e Monochromatica
}

Dulcinéa Gonçalves TEIXEIRA $^{1}$

Maria de Fátima MARTINS²

José Luiz GUERRA ${ }^{3}$

Maria Angélica MIGLINO ${ }^{1}$

\section{Correspondência para:}

DULCINÉA GONÇALVES TEIXEIRA

Departamento de Cirurgia

Faculdade de Medicina Veterinária e

Zootecnia

Universidade de São Paulo

Av. Prof. Dr. Orlando Marques de Paiva, 87

05508-000 São Paulo - SP

dulcinea@usp.br

Recebido para publicação: 17/08/2004 Aprovado para publicação: 13/07/2005

\author{
1 - Departamento de Anatomia Veterinária da Faculdade de Medicina \\ Veterinária e Zootecnia da Universidade de São Paulo, São Paulo - SP \\ 2 - Departamento de Heliciário Experimental "Profa. Dra. Lor Cury" da \\ Faculdade de Medicina Veterinária e Zootecnia da Universidade de São \\ Paulo, Pirassununga - SP \\ 3 - Departamento de Patologia da Faculdade de Medicina Veterinária e \\ Zootecnia da Universidade de São Paulo, São Paulo - SP
}

\section{Resumo}

Este trabalho teve como objetivo fazer um estudo métrico dos segmentos corpóreos do sistema reprodutor das espécies Achatina fulica e Achatina monochromatica, estabelecendo parâmetros comparativos entre ambas, para melhor conhecimento dessas espécies. Foram usados 15 exemplares adultos de cada espécie. Após o processo seletivo, pesagem e tomada das medidas da concha, os espécimes foram sacrificados por congelamento em freezer, a $-2^{\circ} \mathrm{C}$ por aproximadamente $10 \mathrm{~min}$. Retirado o sistema reprodutor, com incisão longitudinal, procedeu-se as medidas de cada segmento. A análise estatística mostrou que, apesar dos espécimes serem mantidos sob as mesmas condições ambientais e alimentares e submetidos ao mesmo tipo de seleção, ocorreram variações no desenvolvimento e tamanho dos órgãos reprodutores. O aspecto morfológico do sistema reprodutor das duas espécies difere macroscopicamente em seus vários segmentos, porém, a disposição e a localização dos mesmos são idênticas.

\section{Introdução}

O escargot é um molusco terrestre, pertencente ao Phyllum Mollusca, ordem Stylommatophora, família Achatinidae, do gênero Achatina ${ }^{1}$. É um animal pulmonado, tem concha espiralada, cabeça distinta com tentáculos e olhos, pé desenvolvido e massa visceral com giro de $180^{\circ}{ }^{2}$, é ovíparo e hermafrodita incompleto e necessita de um parceiro para que haja reprodução ${ }^{3}$. Os escargots, após a cópula, ficam em condições de fazer posturas de ovos fecundados e férteis, e gerar novas progênies ${ }^{4}$. Wanvipa et al..$^{5}$ descrevem o sistema reprodutor da espécie Achatina fulica, como um conjunto de órgãos masculinos representados pela
Palavras-chave: Achatina fulica. Achatina monochromatica. Sistema reprodutor. glândula prostática, ducto deferente, pênis e o saco dardo; e o feminino pela glândula de albúmen, útero, vagina e espermateca. $\mathrm{O}$ ovotestis é o órgão que produz tanto espermatozóides quanto óvulos.

Bequaert $^{6}$, considerando a anatomia externa da espécie Achatina fulica, relata que as conchas, totalmente desenvolvidas, apresentam tamanho médio de $100 \mathrm{~mm}$ a $200 \mathrm{~mm}$ de comprimento. Em relação a sua coloração, apresentam-se fortemente marcadas com listras verticais castanhoescuras ou pretas amarronzadas em um fundo amarelado ou branco-sujo, com pequenas manchas difusas. A subespécie monochromatica, por sua vez, caracteriza sua coloração como uniformemente amarela, 
sem listras escuras, de columela e parede parietal vermelho-vinho e lábio externo branco azulado. $\mathrm{O}$ autor ainda releva a importância de se investigar a anatomia interna das mesmas sob as condições ambientais.

Barnes ${ }^{7}$ menciona que a espécie Achatina monochromatica diferencia-se facilmente das outras por sua concha monocromática, totalmente desprovida de franjas escuras e ondulada, associada à espécie. Uma característica de maior importância é a postura de poucos ovos (4080), quando comparados ao da Acbatina fulica que é de (200-500 em média) e também quanto ao tamanho $(11,3$ e $8,5 \mathrm{~mm}$, respectivamente). O tamanho dos ovos permite obter dados quanto a sua classificação, e são necessários estudos anatômicos para confirmar as descrições de sua concha.

No presente trabalho foi estudada a variação métrica do sistema reprodutor de escargots comparativa ente as espécies Achatina fulica e Achatina monochromatica, com $\mathrm{o}$ intuito de contribuir para os processos de seleção e aperfeiçoamento das linhagens.

\section{Materiais e Métodos}

O processo seletivo foi efetuado separando-se as matrizes adultas mais prolíficas com alto índice de eclodibilidade, que seriam os progenitores das gerações subseqüentes. As matrizes foram separadas, pesadas, mensuradas e acompanhadas suas posturas.

Após o nascimento, cada progênie foi pesada e medida. Do total de progênies, os $50 \%$ maiores e mais pesados foram separados em outra caixa, enquanto que os $50 \%$ restantes foram eliminados.

Assim, o procedimento para seleção foi repetido semanalmente até a fase adulta (doze meses) de modo que foi possível separar os animais maiores que passaram a ser considerados matrizes. Foram escolhidas 15 matrizes de cada espécie pelo programa de melhoramento genético do Heliciário
Experimental, Prof ${ }^{a} \operatorname{Dr}^{a}$. Lor Cury, da Faculdade de Medicina Veterinária e Zootecnia - Campus de Pirassununga - USP.

Os exemplares com um ano de idade das espécies Achatina fulica e Achatina monochromatica foram mantidos cada qual em caixas de madeira de $60 \mathrm{~cm} \times 60 \mathrm{~cm} \times 30 \mathrm{~cm}$. Em cada área de $1 \mathrm{~m}^{2}$ eram mantidos 15 animais. Nesse habitat foram alimentados "ad libitium" com água e ração balanceada preparada no Heliciário Experimental, segundo as normas de Pacheco e Martins ${ }^{8}$.

A massa corpórea foi determinada em balança analítica de precisão $\left(\right.$ Marte $^{\circledR}$ modelo AS $500 \mathrm{n}^{\circ}$ 227619) e as dimensões da concha, em seu comprimento e largura antes da retirada do animal (Figuras 1 e 2), foram medidas com um paquímetro. Os espécimes foram congelados em freezer, a $-2^{\circ} \mathrm{C}$ por aproximadamente $10 \mathrm{~min}$ como forma de sacrifício.

A concha foi quebrada com cuidado e o animal retirado. O sistema reprodutor como um todo foi fotografado (Figura 3) e esquematizado (Figura 4). Para preparar as peças anatômicas, a dissecação foi realizada pela incisão no plano mediano, começando pela boca, seguindo o sentido crânio lateral no antímero esquerdo acompanhando, assim, a torção geral do corpo. Finalmente, seccionou-se o músculo retrator do pênis, liberando todo o conjunto de órgãos reprodutores. Cada segmento, depois de retirado o sistema reprodutor, foi medido, fotografado e acondicionado em recipiente com cloreto de sódio a $0,9 \%$.

\section{Análise estatística}

A análise estatística foi efetuada com o auxílio do Software Graph Pad InStat 3.0 para o teste t de Student e para o coeficiente de correlação de Pearson.

\section{Resultados}

Os valores métricos médios correspondentes a massa do animal vivo, comprimento e largura da concha e dos diversos segmentos do sistema reprodutor 
Tabela 1 - Médias, desvio padrão e coeficiente de variação das medidas de conchas e massa corpórea das espécies Achatina fulica e Achatina monochromática ( $\mathrm{N}=30$ observações -15 de cada espécie). Pirassununga - 2002

\begin{tabular}{|c|c|c|c|c|c|c|c|}
\hline \multirow{2}{*}{ Animal } & \multicolumn{3}{|c|}{ Achatina fulica } & \multirow{2}{*}{ Animal } & \multicolumn{3}{|c|}{ Achatina monochromatica } \\
\hline & $\begin{array}{l}\text { Comprimento } \\
(\mathrm{mm})\end{array}$ & $\begin{array}{l}\text { Largura } \\
(\mathrm{mm})\end{array}$ & $\begin{array}{l}\text { Peso } \\
\text { (g) }\end{array}$ & & $\begin{array}{l}\text { Comprimento } \\
(\mathrm{mm})\end{array}$ & $\begin{array}{c}\text { Largura } \\
(\mathrm{mm})\end{array}$ & $\begin{array}{c}\text { Peso } \\
(\mathrm{g})\end{array}$ \\
\hline 1 & 93,5 & 53,0 & 150,4 & 1 & 119,0 & 56,0 & 204,6 \\
\hline 2 & 94,0 & 51,0 & 135,5 & 2 & 119,0 & 61,0 & 186,7 \\
\hline 3 & 82,0 & 48,5 & 143,0 & 3 & 93,0 & 50,0 & 116,6 \\
\hline 4 & 90,0 & 52,0 & 142,0 & 4 & 95,0 & 45,0 & 105,0 \\
\hline 5 & 81,0 & 51,0 & 121,4 & 5 & 102,0 & 48,0 & 133,9 \\
\hline 6 & 83,0 & 49,0 & 127,3 & 6 & 103,0 & 47,0 & 105,3 \\
\hline 7 & 83,0 & 41,0 & 121,2 & 7 & 96,0 & 46,0 & 100,8 \\
\hline 8 & 88,0 & 48,0 & 125,1 & 8 & 105,0 & 54,0 & 110,9 \\
\hline 9 & 90,5 & 51,5 & 145,7 & 9 & 119,0 & 59,0 & 222,2 \\
\hline 10 & 92,0 & 55,0 & 175,9 & 10 & 93,0 & 45,0 & 94,0 \\
\hline 11 & 84,0 & 49,0 & 122,3 & 11 & 102,0 & 45,0 & 124,5 \\
\hline 12 & 100,0 & 58,5 & 181,9 & 12 & 101,0 & 49,0 & 123,9 \\
\hline 13 & 95,0 & 53,0 & 163,8 & 13 & 103,0 & 50,0 & 113,6 \\
\hline 14 & 105,0 & 55,5 & 170,9 & 14 & 104,0 & 51,0 & 174,9 \\
\hline 15 & 101,5 & 51,0 & 184,1 & 15 & 124,0 & 62,0 & 199,6 \\
\hline Média & 90,8 & 51,1 & 147,4 & Média & 105,2 & 51,2 & 141,1 \\
\hline $\begin{array}{l}\text { Desvio } \\
\text { padrão }\end{array}$ & 7,5 & 4,0 & 22,8 & $\begin{array}{l}\text { Desvio } \\
\text { padrão }\end{array}$ & 10,2 & 5,9 & 43,6 \\
\hline $\begin{array}{c}\text { Coeficiente } \\
\text { de } \\
\text { variação }\end{array}$ & 8,26 & 7,8 & 15,6 & $\begin{array}{c}\text { Coeficiente } \\
\text { de } \\
\text { variação }\end{array}$ & 9,7 & 11,5 & 30,9 \\
\hline
\end{tabular}

de cada espécie estudada, bem como a análise estatística preconizada, encontram-se nas tabelas 1,2,3, e 4 que mostram os valores para a espécie Achatina fulica e Achatina monochromatica.

A extensão do sistema reprodutor é maior na Achatina monochromatica do que na Achatina fulica atingindo, em média, $11,5 \mathrm{~cm}$ na Achatina monochromatica $7,5 \mathrm{~cm}$ na Achatina fulica.

Apesar dos animais terem sido submetidos às mesmas condições ambientais $\mathrm{e}$ alimentares, e terem passado pelo mesmo tipo de seleção, houve variação no seu desenvolvimento e na biometria dos seus órgãos reprodutores.

As médias gerais observadas para as espécies Achatina monochromatica e Achatina fulica quanto ao comprimento da concha foram $105,2 \mathrm{~mm}$ e $90,8 \mathrm{~mm}$, para o peso foram $141,1 \mathrm{~g}$ e $147,4 \mathrm{~g}$, quanto ao comprimento do ovotestis $4,9 \mathrm{~mm}$ e $2,0 \mathrm{~mm}$, quanto à largura da glândula de albúmen de $11,0 \mathrm{~mm}$ e $9,3 \mathrm{~mm}$ e quanto ao ducto deferente $47,1 \mathrm{~mm}$ e $35,7 \mathrm{~mm}$, respectivamente.

No teste $t$ de Student puderam ser observadas diferenças significativas $(\mathrm{P}>0,01)$ para o comprimento da concha, no ovotestis e no ducto deferente entre as duas espécies estudadas.

Para a Achatina fulica as estimativas dos coeficientes de correlação foram altas entre as características avaliadas seguintes: o comprimento da concha e o comprimento da glândula de albúmen $(r=0,70)$; entre o comprimento da concha e oútero $(r=0,74)$; entre o comprimento da concha e a glândula prostática $(\mathrm{r}=0,86)$; entre a largura da concha e o comprimento da glândula de albúmen $(\mathrm{r}=0,76)$; entre a largura da concha e o útero $(r=0,74)$; entre a largura da concha e a glândula prostática ( $\mathrm{r}$ $=0,83$ ); entre a massa in vivo e o ovotestis ( $\mathrm{r}$ 
Tabela 2 - Medidas em milímetros das genitálias, desvio padrão e coeficiente de variação das espécies Achatina monochromática e Achatina fulica ( $N=30$ observações - 15 de cada espécie) - Pirassununga - 2002

\begin{tabular}{|c|c|c|c|c|c|c|c|c|c|c|c|c|c|c|}
\hline \multirow{3}{*}{ Animal } & \multirow{2}{*}{\multicolumn{2}{|c|}{ Ovotestis }} & \multirow{2}{*}{\multicolumn{2}{|c|}{$\begin{array}{c}\text { Ducto } \\
\text { Hermafrodita }\end{array}$}} & \multicolumn{4}{|c|}{ Glândula Albúmen } & \multirow{2}{*}{\multicolumn{2}{|c|}{ Útero }} & \multirow{2}{*}{\multicolumn{2}{|c|}{$\begin{array}{l}\text { Glândula } \\
\text { Prostática }\end{array}$}} & \multirow{2}{*}{\multicolumn{2}{|c|}{$\begin{array}{c}\text { Ducto } \\
\text { Deferente }\end{array}$}} \\
\hline & & & & & \multicolumn{2}{|c|}{ Comprimento } & \multicolumn{2}{|c|}{ Largura } & & & & & & \\
\hline & $1^{*}$ & $2 * *$ & 1 & 2 & 1 & 2 & 1 & 2 & 1 & 2 & 1 & 2 & 1 & 2 \\
\hline 1 & 5,0 & 3,0 & 10,0 & 17,0 & 30,0 & 50,0 & 9,0 & 11,0 & 42,0 & 56,0 & 38,0 & 51,0 & 56,0 & 24,0 \\
\hline 2 & 2,0 & 3,0 & 13,0 & 11,0 & 35,0 & 52,0 & 8,0 & 14,0 & 41,0 & 64,0 & 30,0 & 57,0 & 36,0 & 40,0 \\
\hline 3 & 7,0 & 2,5 & 17,0 & 21,0 & 52,0 & 42,0 & 17,0 & 7,0 & 70,0 & 42,0 & 62,0 & 35,0 & 62,0 & 29,0 \\
\hline 4 & 3,0 & 1,0 & 10,0 & 17,0 & 44,0 & 30,0 & 12,0 & 7,0 & 50,0 & 45,0 & 40,0 & 36,0 & 41,0 & 26,0 \\
\hline 5 & 4,0 & 2,0 & 11,0 & 12,0 & 29,0 & 23,0 & 11,0 & 2,0 & 42,0 & 48,0 & 40,0 & 42,0 & 47,0 & 37,0 \\
\hline 6 & 4,0 & 2,0 & 11,0 & 25,0 & 30,0 & 38,0 & 8,0 & 10,0 & 36,0 & 42,0 & 34,0 & 40,0 & 45,0 & 36,0 \\
\hline 7 & 3,0 & 1,0 & 12,0 & 19,0 & 45,0 & 20,0 & 15,0 & 4,0 & 52,0 & 41,0 & 43,0 & 33,0 & 47,0 & 26,0 \\
\hline 8 & 3,0 & 1,0 & 9,0 & 10,0 & 32,0 & 27,0 & 9,0 & 30,0 & 41,0 & 42,0 & 39,0 & 40,0 & 47,0 & 30,0 \\
\hline 9 & 4,0 & 3,5 & 13,0 & 12,0 & 30,0 & 42,0 & 14,0 & 9,0 & 55,0 & 55,0 & 51,0 & 46,0 & 40,0 & 46,0 \\
\hline 10 & 8,0 & 1,0 & 12,0 & 12,0 & 35,0 & 30,0 & 10,0 & 5,0 & 54,0 & 44,0 & 52,0 & 38,0 & 38,0 & 36,0 \\
\hline 11 & 6,0 & 1,0 & 18,5 & 13,0 & 40,0 & 24,0 & 11,0 & 4,0 & 47,0 & 40,0 & 47,5 & 38,0 & 48,5 & 41,0 \\
\hline 12 & 4,0 & 2,0 & 11,0 & 19,0 & 33,0 & 30,0 & 12,0 & 7,0 & 51,0 & 46,0 & 50,0 & 42,0 & 47,0 & 43,0 \\
\hline 13 & 14,0 & 2,0 & 15,0 & 13,0 & 30,0 & 22,0 & 9,0 & 6,0 & 43,0 & 41,0 & 41,0 & 40,0 & 60,0 & 42,0 \\
\hline 14 & 4,0 & 3,0 & 40,0 & 12,0 & 30,0 & 46,0 & 8,0 & 10,0 & 33,0 & 55,0 & 30,0 & 53,0 & 41,0 & 46,0 \\
\hline 15 & 3,0 & 2,0 & 16,0 & 22,0 & 46,0 & 55,0 & 12,0 & 13,0 & 46,0 & 51,0 & 41,0 & 59,0 & 51,0 & 34,0 \\
\hline Média & 4,9 & 2,0 & 14,6 & 15,6 & 36,1 & 35,4 & 11,0 & 9,3 & 46,7 & 47,5 & 42,6 & 43,3 & 47,1 & 35,7 \\
\hline $\begin{array}{l}\text { Desvio } \\
\text { padrão }\end{array}$ & 3,1 & 0,8 & 7,7 & 4,8 & 7,5 & 11,5 & 2,8 & 6,9 & 9,3 & 7,1 & 8,9 & 8,1 & 7,5 & 6,9 \\
\hline $\begin{array}{c}\text { Coefici- } \\
\text { ente de } \\
\text { variação }\end{array}$ & 62,8 & 42,6 & 53,1 & 30,7 & 20,8 & 32,4 & 25,2 & 74,5 & 19,8 & 14,8 & 20,9 & 18,7 & 16,0 & 19,2 \\
\hline
\end{tabular}

$* 1$ = Achatina monochromatica; ** 2 = Achatina fulica

$=0,80)$; entre a massa in vivo e o comprimento da glândula de albúmen $(\mathrm{r}=$ $0,77)$; entre a massa in vivo e o útero ( $\mathrm{r}=$ $0,84)$ e, por fim, entre a massa in vivo e a glândula prostática $(\mathrm{r}=0,84)$. As demais correlações foram baixas e medianas variando de $-0,07$ a 0,65.

As estimativas dos coeficientes de correlação, para a espécie Achatina monochromatica, foram baixas variando de $\mathrm{r}$ $=-0,45$ a $\mathrm{r}=0,47$.

\section{Discussão}

O estudo do sistema reprodutor das espécies Achatina fulica e Achatina monochromatica reveste-se de importância pela escassez de dados na literatura concernente ao assunto e as implicações de ordem econômica.

Ao considerar a Anatomia Externa da espécie Achatina fulica, Bequaert ${ }^{6}$, relata que a concha, totalmente desenvolvida, apresenta tamanho médio de $100 \mathrm{~mm}$ a $200 \mathrm{~mm}$ de comprimento, todavia, neste trabalho encontrou-se valores entre $93,0 \mathrm{~mm}$ e $124,0 \mathrm{~mm}$. Bequaert ${ }^{6}$ não cita em seu trabalho a idade dos animais com que trabalhou.

Em 1996, Barnes ${ }^{7}$ observou, que a Achatina fulica é considerada uma das maiores espécies e que sua concha pode medir aproximadamente $270 \mathrm{~mm}$ de comprimento. Este valor supera em mais de 100\% a maior medida verificada neste trabalho.

Na literatura não foram encontrados dados 
Tabela 3 - Comparação entre as características avaliadas nas espécies Achatina fulica e Achatina monochromatica por meio do teste 't' de Student. Pirassununga - 2002.

\begin{tabular}{lc}
\hline & 't' observado \\
\hline Comprimento & $4,39^{* *}$ \\
Largura & $0,036^{\text {n.s. }}$ \\
Peso "in vivo" & $0,493^{\text {n.s. }}$ \\
Ovotestis & $3,653^{* *}$ \\
Ducto hermafrodita & $0,48^{\text {n.s. }}$ \\
Comprimento da glândula de albúmen & $0,18^{\text {n.s. }}$ \\
Largura da glândula de albúmen & $0,93^{\text {n.s. }}$ \\
Útero & $0,20^{\text {n.s. }}$ \\
Glândula prostática & $0,25^{\text {n.s. }}$ \\
Ducto deferente & $4,145^{* *}$ \\
\hline
\end{tabular}

Nota: Teste tde Student $(\mathrm{df}=28) ; * \mathrm{P}>0,05=$ Resultado significativo ao nível de $5 \%$ de probabilidade; * $\mathrm{P}>0,01=$ Resultado significativo ao nível de $1 \%$ de probabilidade; ${ }^{\text {ns }} \mathrm{P}<0,05=$ Resultado não significativo ao nível de $5 \%$ de probabilidade.

Tabela 4 - Estimativas dos coeficientes de correlação entre as características avaliadas para a espécie Achatina fulica. Pirassununga - 2002

\begin{tabular}{lccc}
\hline \multicolumn{1}{c}{ Característica } & $\begin{array}{c}\text { Comprimento da } \\
\text { concha }(\mathrm{mm})\end{array}$ & $\begin{array}{c}\text { Largura da } \\
\text { concha }(\mathrm{mm})\end{array}$ & $\begin{array}{c}\text { Peso in vivo } \\
(\mathrm{g})\end{array}$ \\
\hline Ovotestis & 0,61 & 0,65 & 0,80 \\
Ducto hermafrodita & $-0,07$ & $-0,10$ & $-0,14$ \\
Comprimento da glândula de albúmen & $-0,70$ & 0,76 & 0,77 \\
Largura da glândula de albúmen & 0,37 & 0,52 & 0,17 \\
Útero & 0,74 & 0,74 & 0,84 \\
Glândula prostática & 0,86 & 0,83 & 0,84 \\
Ducto deferente & 0,19 & 0,14 & 0,27 \\
\hline
\end{tabular}

A
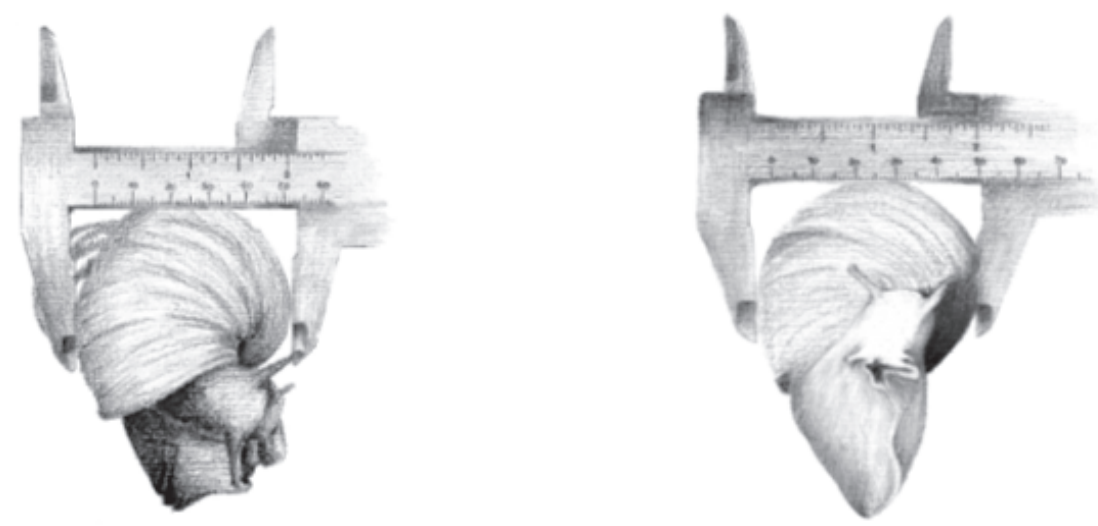

B

Figura 1 - Esquema de mensuração da largura da concha das espécies (A) Achatina fulica e (B) Achatina monochromatica 

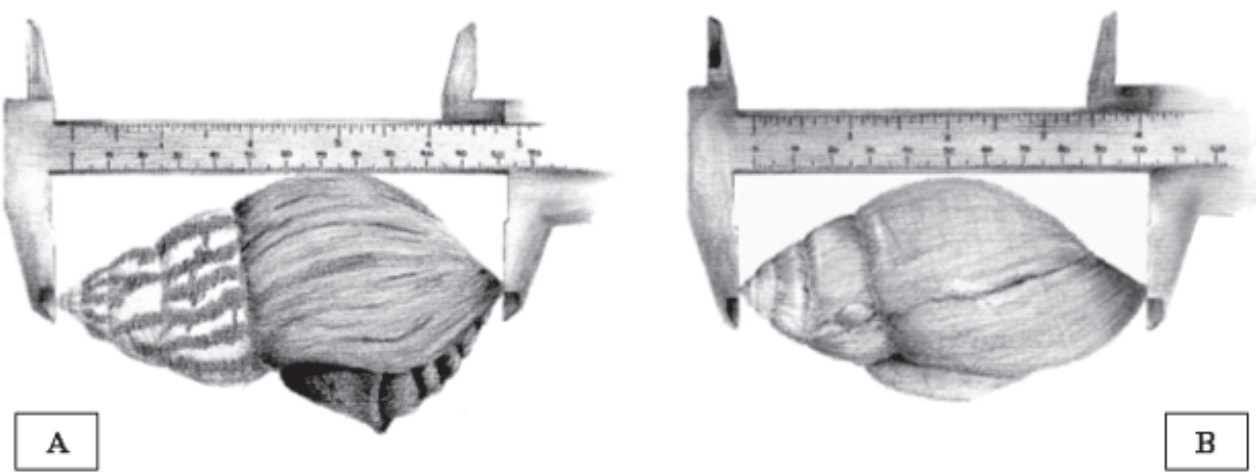

Figura 2 - Esquema de mensuração do comprimento da concha das espécies (A) Achatina fulica e (B) Achatina monochromatica
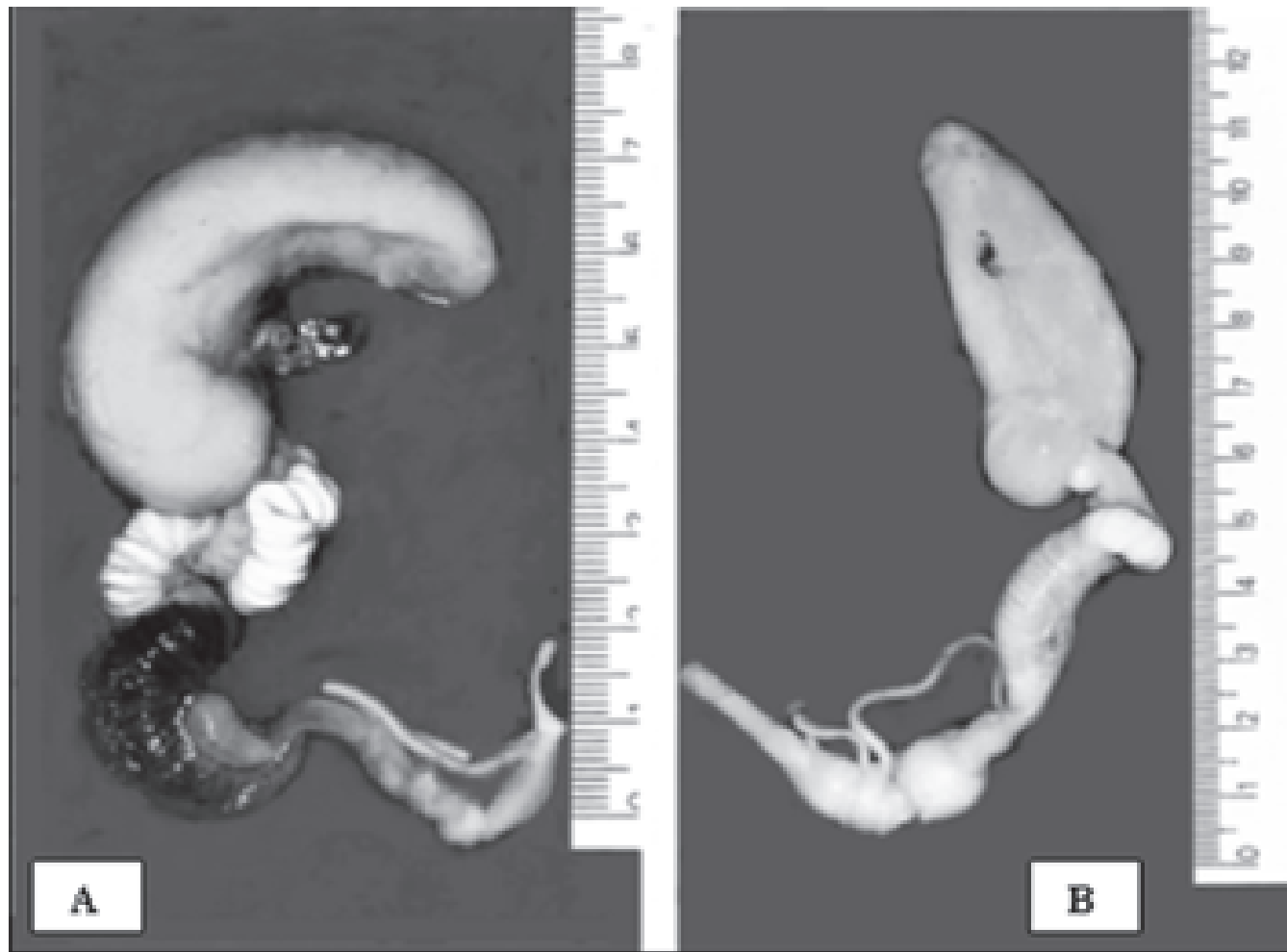

Figura 3 - Fotografia do sistema reprodutor das espécies (A) Achatina fulica-animal número 13 e (B) Achatina monochromatica - animal número 06, em tamanho real $(\mathrm{mm})$ - conforme a Tabela 2

para comparar os valores observados nesta pesquisa para os valores métricos dos segmentos corpóreos para a Achatina monochromatica.

$\mathrm{Na}$ análise estatística a média dos valores métricos dos órgãos do sistema reprodutor das espécies Achatina fulica e Achatina monochromatica mostra diferenças entre o ovotestis, a glândula de albúmen e o ducto deferente. Na Achatina monochromatica o comprimento do ovotestis é maior $4,9 \mathrm{~mm}$, assim como a largura da glândula de albúmen $11,0 \mathrm{~mm}$ e o comprimento do ducto deferente $47,1 \mathrm{~mm}$. Quando comparada com a Achatina fulica, $2,0 \mathrm{~mm}$ para o ovotestis, 


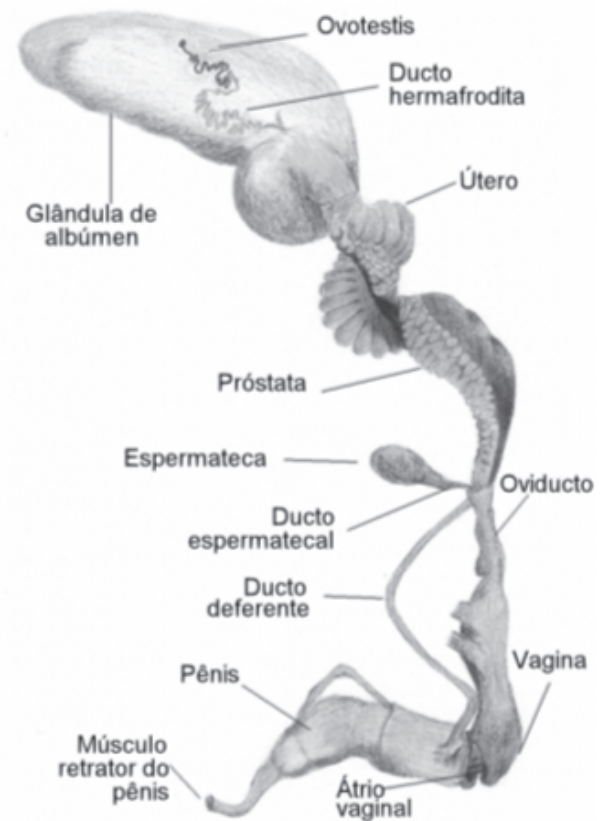

B

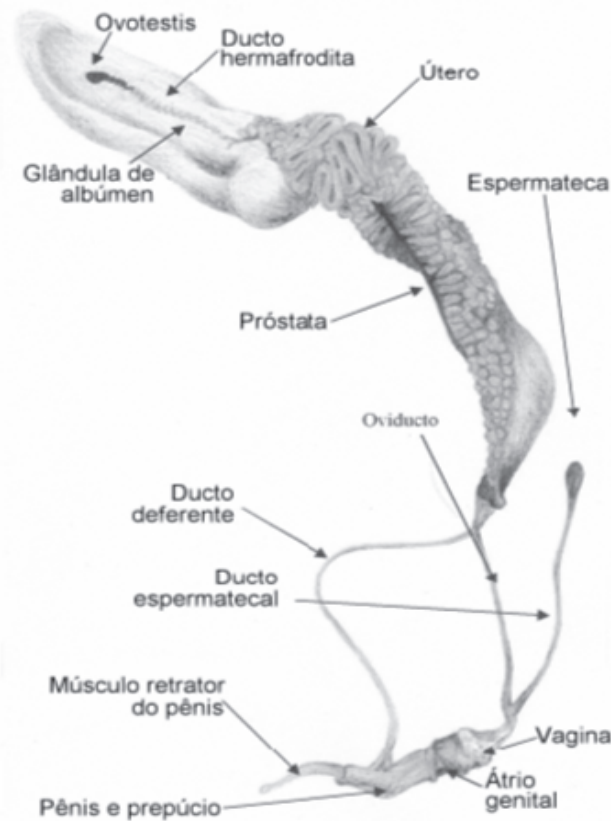

A

Figura 4 - Esquemas representativos do sistema reprodutor das espécies (A) Achatina fulica e(B) Achatina monochromatica.

$9,3 \mathrm{~mm}$ para a largura da glândula de albúmen e $35,7 \mathrm{~mm}$ para o ducto deferente.

Foi possível observar diferenças significativas $(\mathrm{P}>0,01)$, ou seja, o comprimento da concha maior na Achatina fulica (média $=105,2 \mathrm{~mm}$ ), o ovotestis e o ducto deferente maiores na Achatina monochromatica (média $=4,9 \mathrm{~mm}$ e média $=$ 47,1 $\mathrm{mm})$.

As estimativas dos coeficientes de correlação foram, para a espécie Achatina fulica, altas entre os segmentos. Os resultados nas demais correlações apresentaram valores baixos a medianos, entre $-0,07$ e 0,65 . Por outro lado, as estimativas dos coeficientes de correlação foram, para a espécie Achatina monochromatica, baixas tendo um coeficiente de correlação $r^{2}$ entre -0,45 e 0,47. Estas diferenças nas dimensões dos segmentos que compõem o sistema reprodutor da Achatina monochromatica e Achatina fulica talvez sejam uma característica importante quanto a postura ovos; poucos (40-80) na Achatina monochromatica e mais numerosos (em média
200-500) na Achatina fulica bem como relacionados ao tamanho desses ovos (11,3 e $8,5 \mathrm{~mm}$, respectivamente). O tamanho dos ovos permite obter dados quanto a classificação destes animais e são necessários estudos fisiológicos para avaliar se existe relação entre o tamanho do animal e a ovoposição destas espécies.

\section{Conclusão}

Conclui-se que as duas espécies, mesmo submetidas às mesmas condições ambientais e de alimentação, desenvolvemse em ritmos diferentes, ocorrendo variação na biometria de seus órgãos reprodutores (conforme comunicação verbal com mantenedores do Hiliciário a cerca do processo de seleção dos animais para matrizes). O comprimento do ovotestis, a largura da glândula de albúmen e o comprimento do ducto deferente são maiores na Achatina monochromatica do que na Achatina fulica. O útero é anatomicamente 
semelhante nas duas espécies e a vagina apresenta diferenças marcantes quanto ao tamanho e espessura da parede, e é maior e mais espessa na Achatina monochromatica.

O comprimento da concha, do ovotestis e do ducto deferente apresentou diferenças significantes entre as duas espécies $(\mathrm{P}>0,01)$.

\section{Agradecimentos}

Os autores agradecem aos professores da Faculdade de Medicina Veterinária e Zootecnia da Universidade de São Paulo, SP, Dr. Francisco J.H. Blazquez pelas sugestões e ao Sr. Fábio de M. Francisco, graduando da FMVZ-USP, que elaborou os desenhos ilustrativos das peças anatômicas.

\title{
Statistical considerations about the metric values of the corporeal segments of the reproductive system of escargots of the species Achatina fulica and Achatina monochromatica
}

\begin{abstract}
The objective of this work was to perform a metric study of the reproductive system of the species Achantina fulica and Achatina monochromatica, establishing comparative parameters between both, to improve the knowledge of the species. After the selective process, weighting and measures of the shell, the specimens were frozen in freezer to $-2^{0} \mathrm{C}$ for approximately $10 \mathrm{~min}$ as a sacrificing form. For each segments the measures proceeded after the retrieval of the reproductive system through a longitudinal incision. The statistical analysis showed that, in spite of the specimens be maintained under the same environmental and alimentary conditions and submitted to the same selection type, variations happened in the development and size of the reproductive organs. The morphologic aspect of the reproductive system in both species differs macroscopically in several segments; however, the disposition and the location of those segments are identical.
\end{abstract}

\section{Referências}

1 RUPPERT, E. E.; BARNES, R. D. Zoologia dos invertebrados. 6. ed. São Paulo: Roca, 1996. p. 332352.

2 PACHECO, P.; MARTINS, M. F. Criação e processamento de escargot. São Paulo: 1997. p. 31. Apostila de curso.

3 VIEIRA, M. I. Escargot: criação caseira e comercial. 2. ed. São Paulo: Nobel, 1984.

4 MARTINS, M. F; PACHECO, P. Desempenho reprodutivo do escargot Achatina fulica resultados preliminares. In: CONGRESSO BRASILEIRO DE ZOOLOGIA, 22. Recife. Anais... [S.I.: s.n], 1998. p. 386.

5 WANVIPA, K.; KRUATRACHUE, M.; SUCHART, E. U.; CHITRAMVONG, Y.; SETARUGSA, P.; CHAVADEJ, J.; SOBHON, T. M.; PRASERT, S. Comparative Studies on reproductive systems of Achatina fulica, Hemiplecta distincta and Cyclophorus
Key-words:

Achatina fulica.

Achatina monochromatica. Reproductive system. aurantiacus. Journal of Science Society of Thailand, v. 15, p. 71-107, 1989.

6 BEQUAERT, J. C. Studies in the Achatinidae, a group of African land snails. Bulletin of the Museum of Comparative Zoology at Harvard College. v. 105 n. 1, p. 1-216, 1950.

7 BARNES, R. D. Os moluscos. In: Z_ Zoologia dos invertebrados. 5. ed. São Paulo: Roca, 1996. p. 339496.

8 PACHECO, P.; MARTINS, M. F. O escargot. Revista Higiene Alimentar. V.12-55. p.19-10, 1998. 